\title{
Modeling and Analyzing Taxi Congestion Premium in Congested Cities
}

\author{
Changwei Yuan, ${ }^{1}$ Dayong Wu, ${ }^{2}$ Dali Wei, ${ }^{3}$ and Hongchao Liu ${ }^{2}$ \\ ${ }^{1}$ School of Economy and Management, Chang'an University, Xian, Shaanxi 710064, China \\ ${ }^{2}$ Department of Civil, Environmental and Construction Engineering, Texas Tech University, Lubbock, TX 79409, USA \\ ${ }^{3}$ Partners for Advanced Transportation Technologies, University of California, Berkeley, 1357 S 46th St, Richmond, CA 94804, USA
}

Correspondence should be addressed to Dayong Wu; jasond.wu@ttu.edu

Received 22 November 2016; Revised 2 February 2017; Accepted 19 February 2017; Published 19 March 2017

Academic Editor: William H. K. Lam

Copyright (C) 2017 Changwei Yuan et al. This is an open access article distributed under the Creative Commons Attribution License, which permits unrestricted use, distribution, and reproduction in any medium, provided the original work is properly cited.

\begin{abstract}
Traffic congestion is a significant problem in many major cities. Getting stuck in traffic, the mileage per unit time that a taxicab travels will decline significantly. Congestion premium (or so-called low-speed fare) has become an increasingly important income source for taxi drivers. However, the impact of congestion premium on the taxicab market is not widely understood yet. In particular, modeling and analyzing of the taxi fare structure with congestion premium are extremely limited. In this paper, we developed a taxi price equilibrium model, in which the adjustment mechanism of congestion premium on optimizing the taxi driver's income, balancing the supply and demand, and eventually improving the level of service in the whole taxicab market was investigated. In the final part, we provided a case study to demonstrate the feasibility of the proposed model. The results indicated that the current taxi fare scheme in Beijing is suboptimal, since the gain from the raise of congestion premium cannot compensate for the loss from the demand reduction. Conversely, the optimal fare scheme suggested by our model can effectively reduce the excessive demand and reach the supply-demand equilibrium, while keeping the stability of the driver's income to the maximum extent.
\end{abstract}

\section{Background}

Taxi fares generally include a flag-down fare, a fare charged by the ride length, congestion premium (or so-called lowspeed fare, which is the cost of wait upon request of the passenger), and other expenses (such as fuel surcharges). As a special component in the taxi fare structure, congestion premium accounts for the extra operational cost for a taxicab in congested traffic. In recent years, traffic congestion is getting worse and worse in many cities, especially in some megacities (e.g., Beijing, a city with 21 million people and 5 million vehicles) $[1,2]$. The mileage per unit time that a taxicab travels declines rapidly and congestion premium has therefore become an increasingly important source of income for taxi drivers in congested cities.

However, little insight has been gained into the mechanism of congestion premium in tuning the taxi driver's income, although it has been implemented as a common practice for a long time. With lack of a systematic understanding, it is always a blinding decision-making process for the government to make a policy to either raise or lower congestion premium, and hence the policy cannot guarantee the balance between the supply side (or taxi drivers) and the demand side (or taxi passengers). As a result, many current fare policies can neither compensate for the additional cost caused by long-period traffic jams nor adjust the taxi demand effectively. On the contrary, the policies can easily lead to service refusal, since being trapped in congested traffic for a long time usually reduces the driver's income substantially and congestion premium cannot effectively offset the loss of the driver's income. In some extreme cases, for example, during peak hours, taxi drivers prefer being out of service than working inside the city, which in turn exacerbates the imbalance between the supply and demand in the taxicab market. A recent survey in Beijing found that as many as nearly 10,000 taxi vehicles were at rest during the afternoon peak hours, evading service in congested hours [2-4]. Thus, it is very necessary to analyze the adjustment mechanism of congestion premium in congested cities. 
In this study, we attempted to develop a taxi price equilibrium model with consideration of congestion premium and to explore an adjustment mechanism for optimizing the taxi driver income, balancing the supply and demand, and eventually improving the service level of the whole taxi market. The proposed model can be deemed as a new and original idea in modeling the taxi market system and as a useful supplement to enhance the body of the existing literature.

The rest of the paper is organized as follows. Section 2 performs a critical and thorough literature review on the models of taxi market system. By comparing with existing studies, the contributions of this study have been also highlighted in this section. Section 3 discusses how to build a taxi price equilibrium model with congestion premium. Section 4 conducts a detailed analysis of the proposed model. Two critical congestion premium fares have been identified. Section 5 focuses on finding the optimal congestion premium and the solution for a desired income. Three cases are identified under various scenarios with different parameter combinations. Section 6 provides a case study in Beijing's taxi market to demonstrate the property of this model. Section 7 concludes the paper with major findings.

\section{Literature Review}

2.1. Related Works on Modeling Taxi Market. Taxi is an active mode of transportation whose flexible service is irreplaceable in most urban areas. How to provide a speedy, comfortable, and efficient taxi service through the regulation has grown immensely as a subject of city government concern. Generally, Taxi regulations are mainly concerned with two primary issues (price and entry controls) and many models have been extensively developed to tackle them. Although each model is analyzed from different points of view, the models to date can be grouped into two categories: aggregated models (e.g., [5-14]) and equilibrium models (e.g., a series of models presented by Yang and Wong during the period of 1997-2010 by studying the taxicab market in the network of Hong Kong) [15-23].

Aggregated models are developed mainly based on an abstract, aggregated demand, and supply model or based on a simplified, specific structural model of either a dispatching or cruising taxi service in either competitive or monopolistic markets [24]. Analytical economic analysis can be conducted to derive some descriptive conclusions concerning the consequence of taxi regulation [25]. In 1972, Douglas constructed the first aggregated model of taxi price and believed that the free market competition cannot form an effective taxi price, and hence the taxi price should be regulated. All the later authors in this group used the model proposed by Douglas for developing their models and examined them with different market configurations. Each model has added its value to the evolution of the taxicab market modeling. De Vany (1975) added an index of the full prices to the calculation of the demand. Manski and Wright (1976) assumed a Poisson process of customer arrivals in a FIFO queue discipline for the rank market. Daniel (1978) used a demand function depending on the number of vacant taxis and the price. Cairns and Liston-Heyes (1996) supposed uniform demand within the day decreasing with the increase of the waiting time. Chang and Chu (2009) used log-nonlinear and log-linear functions, respectively, for simulating demand. Von Massow and Canbolat (2010) developed a double queue model simulating a dispatching market, where drivers are assigned to queues in zones and high demand points. The above models calculated total demand and supply and tested them in different markets using different parameters. The principal assumptions underlying the models are the relation between the waiting time and the total number of vacant taxi hours, constant operating cost per hour, and demand estimation based on fares and waiting time of passengers.

However, the supply and demand relationships for taxi services are much more complicated than the models in classical economic analyses. Modeling more realistic demand for and supply of taxi services should take into account road network spatial structure and dynamic origin-destination (O-D) demand pattern [26]. To this end, Yang and Wong developed a series of equilibrium models during the years 1997 to 2010 by studying the taxicab market in the network of Hong Kong, which are deemed more realistic than the aggregated ones. In 1998, Yang and Wong (1998) developed their first equilibrium model by using the model of Douglas (1972) in an origin-destination matrix, where demand is fixed for each pair of O-D. Along this line, the supply-demand equilibrium and the relation between the fleet size, elastic demand, and taxi fare have been well analyzed by them. They introduced distance-based and delay-based taxi fare models that take into account the effects of traffic congestion on the taxi market $[19,21]$. The road network, the origin-destination based demand, and the search friction were modeled by [22, $25,27]$. Taking into account the spatial structure of the taxi market, their models are capable of quantifying a number of system performance measures such as utilization rate and level of service quality for different zones. Recently, Yang et al. (2010b, 2010c) further improved the model by considering the bilateral waiting and search behaviors, and more realistic waiting time functions were developed.

It is noticeable that taxi models are getting more and more realistic as time goes. The first group of models used aggregated demand and supply values, lacking the consideration of traffic impacts on the taxicab market. The second group's researchers improved the model by introducing roadway network spatial characteristics and dynamic trip generationdistribution and assignment. They made a lot of efforts on introducing the rules (e.g., the network knowledge, the learning process of taxi drivers, and the customer-driver function) to simulate the taxicab market more realistically. In the opinion of the authors, each group of models has its own merits and can be used in different level of scales. Aggregated models can use fewer variables to catch major behaviors of taxicab market and explain fare and entry regulations more clearly, while equilibrium models can simulate the taxicab market more in detail by taking into account traffic impacts (spatial variables of supply and demand, dynamic trip generation and assignment, the congestion, etc.). However, more detailed models usually require more detailed data which are more difficult to collect. In this regard, data availability has become more important issue than ever. 
Thanks to the development of new advanced intelligent transportation systems (ITS) and other technologies (e.g., Big Data, cloud and distributing computing, and Machine Learning), a lot of data can be recorded and stored for analyzing, and more detailed and complex models can be developed $[28,29]$. The authors would like to call them the third group of taxi models: data-driven models. Some researchers have already conducted some very interesting work in this field [30-34]. Notably, the NYC taxi data has been used widely in these studies, since it is one of the largest available open-source taxi datasets in the world.

2.2. The Contributions of This Study. In previous studies, a common assumption is that the taxi market is well regulated and little attention has been paid to the issue of service refusal as well as its impact on the supply-demand equilibrium. For example, in the multiperiod dynamic model proposed by Yang et al. (2005b), the service intensity was treated as an endogenous variable, which was determined through finding the equilibrium state in which drivers cannot alter their work schedules to increase their profits. Because of the competition, the net profits of drivers in service and out of service are both zero in the period when the number of taxis in service is less than the fleet size. It is theoretically sound but hard to justify in real-world situation, where the profit for drivers in service is obviously positive; otherwise they will not choose to serve. The most important reason why the driver evades service during the peak hour (or in congested traffic) is the lower-than-expected profit. The drivers often complained that the profit is too low and the level of stress is high when driving in congested traffic. As a result, the drivers would rather suspend the service and take rests (or the so-called service refusal phenomenon). In some extreme cases, that is, during the peak hours, taxi drivers prefer being out of service than working inside the city, which in turn exacerbates the imbalance between the supply and demand in the taxi market. A recent survey in Beijing found that as many as nearly 10,000 taxi vehicles were at rest during the afternoon peak hours, evading service in congested hours. However, this idling-type service refusal is legitimate; and, therefore, city government cannot apply punitive policies or coercive measure to eliminate this service refusal phenomenon. In this regard, the government's focus has been directed toward motivating and stimulating taxi drivers back to service by regulating the market through fare adjustment [35].

Studies to date have mostly focused on well-regulated markets, where service refusal is not present or can be negligible. Little insights have been gained into the mechanism of congestion premium in tuning the income of taxi driver and correspondingly reducing this unpleasant refusal phenomenon, although congestion premium has been implemented as a common practice for a long time in congested cities. In lacking of a systematic understanding of the interactions between service refusal and congestion premium, as well as its impact on the optimal and maximum profit of taxi services, making a policy to either raise or lower congestion premium will be always a blinding decision-making process for the city government. Therefore, the implemented fare policies usually cannot guarantee the balance between both sides (the supply side or taxi drivers and the demand side or passengers). As a result, many current taxi fare schemes can neither compensate for the additional cost caused by longperiod traffic jams nor adjust the demand effectively in the market to improve the level of taxi service. On the contrary, they can easily lead to service refusal, since being trapped in congested traffic for a long time usually hurts the driver's income substantially.

In this study, we made an attempt to construct a taxi price equilibrium model with congestion premium and explore its adjustment mechanism in optimizing the taxi driver's income, balancing the supply and demand in the taxi market, and eventually improving the service level of taxi market at the aggregated level. The proposed price model can be considered as a new and original idea in the taxi modeling and as a useful supplement to enhance the body of the existing literature. An illustrative case study was also provided using the data of Beijing's taxicab market to demonstrate the model's feasibility.

\section{A Taxi Price Equilibrium Model with Congestion Premium}

In this section, we propose a taxi price equilibrium model to describe the dynamic interactions among congestion premium, demand, revenue, and operation cost. The proposed model will provide a mathematical framework to analyze the impact of congestion premium on the taxi market. Major variables used in the paper are defined in Notations.

Normally, the taxi fare consists of an initial flag-fall charge and a variable component accrued at a constant per length unit. As a common practice in China, especially in large cities, congestion premium is also included in the fare structure. However, this fare is only charged when the speed is less than a certain threshold, $u_{0}$ (in Beijing, $u_{0}$ is $12 \mathrm{~km} / \mathrm{h}$, for example). Hence, for a single trip, the total ride price is formulated as

$$
p\left(p_{t}, t_{c}\right)=p_{s}+p_{l} L+p_{t} t_{c},
$$

where $p_{s}$ is initial flag-fall charge $(\mathrm{CNY}), p_{l}$ is fare charge per occupied unit distance $(\mathrm{CNY} / \mathrm{km}), p_{t}$ is fare charge per waiting time unit (CNY/hour) or congestion premium, $L$ is average ride length $(\mathrm{km})$, and $t_{c}$ is average time in congested traffic (hour).

To distinguish the congested and free-flow traffic condition, taxi trips are categorized into peak and off-peak hour trips. For the peak-hour trip, if traffic on a certain portion of the trip is congested and the corresponding traveling speed is below the threshold of $u_{0}$, congestion premium will be charged along these sections of the trip. For the off-peak-hour trip, taxicabs travel with the free-flow speed along most parts of the trip. Small amounts of stop and go traffic situation can be ignorable. Therefore, for simplicity, only the initial flagdown fare and the fare charged by the ride length are taken into account for the taxi service operated in the off-peak traffic.

Next, we will establish the taxi driver's income for both peak and off-peak hours by considering the elastic demand regarding congestion premium. Let $u_{c}$ be the average travel 
speed for the congested sections along the route during the peak hour; the waiting time in congested traffic $t_{c}$ can be calculated as

$$
t_{c}=\frac{\lambda L}{u_{c}}
$$

where $\lambda$ is the proportion ratio of congested sections.

The demand per unit time (or average trips per hour) $D_{c}$ is assumed to be a decreasing function of the trip fare and the expected waiting time $W_{c}$ for a taxi service given as

$$
D_{c}=g_{D_{c}}\left(p_{t}, t_{j}, W_{c}\right)=g_{D_{c}}\left(p_{t}, \alpha, u_{c}, W_{c}\right) \text {. }
$$

(It should be noted that the waiting times of taxis and passengers are essential in describing the market equilibrium. However, in congested traffic, as often observed, the waiting time for a taxi to meet a customer tends to be zero. Even if the search friction indeed exists, its impacts are almost ignorable in congested traffic.) Since this paper puts focus on the analysis of congestion premium, $p_{s}$ and $p_{l}$ are viewed as parameters instead of the variables for $D_{c}$.

Now let us derive the expected waiting time for a customer. If the demand during the off-peak hours is larger than the taxi fleet size $N$, only a proportion of the demand can be served. Let $N_{o, c}$ denote the number of occupied taxis and let $N_{v, c}$ denote the number of vacant taxis. Clearly, we have

$$
\begin{aligned}
& N_{o, c}=\min \left(N, D_{c} T_{c}\right), \\
& N_{v, c}=N-N_{o, c},
\end{aligned}
$$

where $T_{c}$ is the average ride time as defined by

$$
T\left(\lambda, u_{c}\right)=L\left(\frac{\lambda}{u_{c}}+\frac{1-\lambda}{u_{f}}\right) .
$$

The waiting time $W_{c}$ is a decreasing function of the number of vacant taxis:

$$
W_{c}=w_{c}\left[N-\min \left(N, D_{c} T_{c}\right)\right] .
$$

Taking (3) and (5) into (6), we have

$$
W_{c}=w_{c}\left(N-\min \left(N, g_{D_{c}}\left(p_{t}, \lambda, u_{c}, W_{c}\right) T_{c}\left(\lambda, u_{c}\right)\right)\right)
$$

Hence, the income of the taxi fleet per hour is

$$
I_{c}=\min \left(N, g_{D_{c}}\left(p_{t}, \lambda, u_{c}, W_{c}\right) T_{c}\left(\lambda, u_{c}\right)\right) p\left(p_{t}, t_{j}\right) .
$$

Using the exponential response model proposed by Wong et al. [36], the free-flow demand $D_{f}$ is described by

$$
D_{f}=\widetilde{D}_{f} e^{-\sigma\left(v_{1} p_{s}+v_{2} p_{l} L\right)}
$$

in which $\widetilde{D}_{f}$ is the potential demand of free-flow trips. $L$ is designated as the average trip length. The parameter $\sigma$ is the sensitivity coefficient to the trip cost, while $v_{1}$ and $v_{2}$ are sensitivity parameters to the starting fare and fare per length unit, respectively. $\sigma, v_{1}$, and $v_{2}$ are nonnegative values. Higher riding fares would make more customers turn to other transportation modes and the demand would drop accordingly.

When the demand is larger than the capacity of the taxi fleet, only a proportion of the demand can be served, so a capacity constraint needs to be imposed to calculate the realized demand trips. Let the length of the off-peak hours be $P_{f}$ and the average trip time for the free-flow demand is

$$
t_{f}=\frac{L}{u_{f}} .
$$

Hence, the realized demand in off-peak hours $D_{f r}$ is

$$
D_{f r}= \begin{cases}\widetilde{D}_{f} e^{-\sigma\left(v_{1} p_{s}+v_{2} p_{l} L\right)} & \widetilde{D}_{f} e^{-\sigma\left(v_{1} p_{s}+v_{2} p_{l} L\right)}<\frac{N P_{f}}{t_{f}} \\ \frac{N P_{f}}{t_{f}} & \text { otherwise, }\end{cases}
$$

where $N$ is the fleet size. With the realized demand, the income during the off-peak hours is simplified as

$$
I_{f}=D_{f r}\left(p_{s}+p_{l} L\right) \text {. }
$$

During peak hours, a proportion of the trip goes through congested road sections. The speed along this section of the trip is less than the threshold of $u_{0}$, which would trigger congestion premium in the fare structure. Similar to the demand in off-peak hours, the demand of trips for the peak hours is

$$
D_{c}=\widetilde{D}_{c} e^{-\sigma\left(v_{1} p_{s}+v_{2} p_{l} L+v_{3}\left(L \lambda / u_{c}\right) p_{t}\right)}
$$

where $\sigma, v_{1}$, and $v_{2}$ are the same sensitivity parameters as defined in (3). $v_{3}$ is the sensitivity parameter for congestion premium. $\lambda$ is the congested proportion of the trip, in which the average traveling speed $u_{c}$ is less than the threshold value of $u_{0}$. The demand given in (13) also needs to be constrained by the capacity of the taxi fleet. The average trip time for the congested demand is given by

$$
t_{c}=\frac{(1-\lambda) L}{u_{f}}+\frac{\lambda L}{u_{c}},
$$

where $u_{f}$ is the free-flow speed.

Letting the length of the peak hours be $P_{c}$, the maximum number of trips that can be served by the taxi fleet with a size of $N$ is

$$
D_{c m}=\frac{N P_{c}}{t_{c}}
$$

(Note that $D_{c m}$ is referred to as the realized demand during peak hours, which is assumed to be only dependent on the fare. It is not the maximum potential demand in the market.) Combining (13) and (15), the realized demand in peak hours $D_{c r}$ is 


$$
D_{c r}= \begin{cases}\widetilde{D}_{c} e^{-\sigma\left(v_{1} p_{s}+v_{2} p_{l} L+v_{3}\left(L \lambda / u_{c}\right) p_{t}\right)} & \widetilde{D}_{c} e^{-\sigma\left(v_{1} p_{s}+v_{2} p_{l} L+v_{3}\left(L \lambda / u_{c}\right) p_{t}\right)}<D_{c m} \\ D_{c m} & \text { otherwise }\end{cases}
$$

With the developed demand equations in both peak and offpeak hours, the average income of a taxi driver can be derived as

$$
=\frac{\left[p_{s}\left(D_{f r}+D_{c r}\right)+p_{l}\left(D_{f r}+D_{c r}\right) L+\left(D_{c r} \lambda L / u_{j}\right) p_{t}\right]}{N} .
$$

Equation (17) establishes a taxi driver's income structure, where congestion premium tunes the income by affecting both the trip demand and the fare per trip.

Part of the income has to cover the operational cost, which mainly includes the license fee and the fuel cost. As a common practice in China, taxi fleets are owned by private companies. Each individual driver has to pay her/his company a certain amount of license fee per month. The fuel cost is assumed to be a constant per unit of time. Hence, the operational cost is described by

$$
C=c_{g} T+Q
$$

where $c_{g}$ is the fuel cost per unit of time, $T$ is the working hours per day, and $Q$ is the license fee per day.

The equilibrium is reached when the income is larger than the cost by the amount of expected daily profit as

$$
C+R \leq I
$$

where $R$ is the expected profit.

Congestion premium plays an interesting role in the price equilibrium model through dynamically interacting with the demand and the income. From the perspective of demand, when increasing congestion premium, fewer customers would choose the taxi in congested hours and the demand decreases correspondingly. However, the raise of congestion premium would bring about an increase of the income per trip, which compensates for the loss from the decreased demand. The equilibrium of (19) describes the situation when the driver's expected profit is reached by setting the appropriate congestion premium fare to balance the decrease of demand and the increase of the fare per trip.

\section{Analysis of the Equilibrium Model: Two Critical Congestion Premium Fares}

As pointed out in the previous section, congestion premium tunes the driver's income with the opposite effect on the demand and the fare per trip. An increase in congestion premium would lead to a decreased demand but an increased fare per trip. The combinational effect is analyzed in this section with the particular focus on how the income changes with congestion premium given different sets of parameters.
The analysis offers a systematic understanding of the income model built in (17), which also lays a basis for solving congestion premium given a desired profit.

The income in (17) can be written as a function of congestion premium:

$$
I\left(p_{t}\right)=I_{f}+p_{s} D_{c r}+p_{l} D_{c r} L+\frac{\left(D_{c r} \lambda L / u_{j}\right) p_{t}}{N},
$$

where $I_{f}$ is a constant that is independent of $p_{t}$. Obviously, (20) may not be a continuous function, since the realized trip $D_{c r}$ is a piecewise-defined function. If the demand is below the capacity of $D_{c m}$, the realized demand during peak hours $-D_{c r}-$ equals the demand given by (13). Otherwise, it equals a constant capacity of $D_{c m}$. To relate congestion premium to these two cases, (16) is rewritten as

$$
D_{c r}= \begin{cases}\widetilde{D}_{c} e^{-\sigma\left(v_{1} p_{s}+v_{2} p_{l} L+v_{3}\left(L \lambda / u_{c}\right) p_{t}\right)} & p_{t}>p_{t, 1} \\ D_{c m} & 0<p_{t} \leq p_{t, 1}\end{cases}
$$

where $a=v_{1} p_{s}+v_{2} p_{l} L, \phi=\lambda / u_{j}$, and $p_{t, 1}$ is given by

$$
p_{t, 1}=\frac{\left[-\ln \left(D_{c m} / \widetilde{D}_{c}\right) / \sigma-a\right]}{v_{3} \phi L} \text {. }
$$

By introducing $p_{t, 1},(20)$ can be rewritten as

$$
\begin{aligned}
& I\left(p_{t}\right) \\
& = \begin{cases}I_{f}+\frac{P_{c}}{t_{c}}\left(p_{s}+p_{l} L+\phi L p_{t}\right) & p_{t} \leq p_{t, 1} \\
I_{f}+\frac{\widetilde{D}_{c} e^{-\sigma\left(a+v_{3} \phi L p_{t}\right)}\left(p_{s}+p_{l} L+\phi L p_{t}\right)}{N} & p_{t}>p_{t, 1} .\end{cases}
\end{aligned}
$$

Correspondingly, the derivative $I^{\prime}\left(p_{t}\right)$ is given as follows:

$$
\begin{aligned}
& I^{\prime}\left(p_{t}\right) \\
& = \begin{cases}\frac{\phi L P_{c}}{t_{c}} & p_{t} \leq p_{t, 1} \\
\frac{\phi L \widetilde{D}_{c} e^{-\sigma\left(a+v_{3} \phi L p_{t}\right)}\left[1-v_{3} \sigma\left(p_{s}+p_{l} L+\phi L p_{t}\right)\right]}{N} & p_{t}>p_{t, 1} .\end{cases}
\end{aligned}
$$

For the second case $\left(p_{t} \leq p_{t, 1}\right)$, it is easy to identify that if there may exist a stationary point:

$$
p_{t, 2}=\frac{\left(1 / v_{3} \sigma-p_{s}-p_{l} L\right)}{(\phi L)}
$$

When $p_{t}$ is less than $p_{t, 1}$, the derivative given by the second equation in (24) is larger than zero; the income grows linearly with an increase in congestion premium. If $p_{t}$ falls between 
$p_{t, 1}$ and $p_{t, 2}$, the income still grows but with a $p_{t}$-dependent gradient. When $p_{t}$ is larger than $p_{t, 2}$, the income decreases with an increase in congestion premium.

However, if $p_{t, 1} \geq p_{t, 2}$, the function of $I\left(p_{t}\right)$ can only be divided into two segments. When $p_{t} \in\left[0, p_{t, 1}\right]$, the income grows linearly with an increase of congestion premium and the gradient is $b P_{c} / t_{c}$. If $p_{t}$ is larger than $p_{t, 1}$, the income decreases with an increase of congestion premium.

It can be seen that $p_{t, 1}$ and $p_{t, 2}$ are two critical values in the system. From the perspective of capacity constraint, $p_{t, 1}$ defines a threshold value that once congestion premium is less than a certain amount, the demand is higher than the capacity, and hence the realized trip is always equal to the capacity. In terms of the demand and the income, $p_{t, 2}$ is a critical value for the situation where raising congestion premium cannot accordingly increase the income due to the decreased demand. Different parameters result in different values of $p_{t, 1}$ and $p_{t, 2}$, while also leading to various forms of the income curve with respect to congestion premium.

\section{Finding the Optimal Income and the Solution of Congestion Premium to a Desired Income}

As analyzed in Section 3, the income has a unique relation with congestion premium given a set of parameters, which can be characterized by two critical points of $p_{t, 1}$ and $p_{t, 2}$. In this section, the analysis will focus on finding the optimal income (upper bound) given $p_{t} \geq 0$ and the solutions of $p_{t}$ if a certain amount of profit is required.

5.1. The Upper and Lower Boundaries. Previous analysis demonstrates combined effects of the dynamic interaction between the customer and the driver through the adjustment of congestion premium. However, due to the capacity constraint, the resulting income function is shown to be firstorder discontinuous in a truncated form.

Before deriving the upper boundaries that are varying in different cases, the lower bound is presented at first, since it is simple and unified for different cases. By taking the limit of (20) based on LHôpital's rule, the result of the limitation is found to be $I_{f}$ derived as given in (26). This is easy to understand, since the demand almost drops to zero if congestion premium of $p_{t}$ tends to positive infinity.

$$
\begin{aligned}
I_{\min }= & I_{f} \\
& +\lim _{p_{t} \rightarrow \infty}\left[\frac{\widetilde{D}_{c} e^{-\sigma\left(a+v_{3} \phi L p_{t}\right)}\left(p_{s}+p_{l} L+\phi L p_{t}\right)}{N}\right] \\
= & I_{f}+\lim _{p_{t} \rightarrow \infty}\left[\frac{\widetilde{D}_{c} \phi L}{\sigma v_{3} \phi L e^{\sigma\left(a+v_{3} \phi L p_{t}\right)} N}\right]=I_{f} .
\end{aligned}
$$

For the upper boundary or the optimal income, three cases are identified under various scenarios with different parameter combinations. To offer a better illustration, two kinds of diagrams are introduced below. One is the $p_{t}$-demand diagram, which describes how the demand changes with congestion premium as well as the impact of the capacity constraint. The other one is the $p_{t}$-income diagram, which shows how the income changes with congestion premium. Two critical congestion premium fares are also highlighted in the diagrams.

Case $1\left(p_{t, 2} \geq p_{t, 1}\right.$ and $\left.p_{t, 2}>0\right)$. In this case, the stationary point $p_{t, 2}\left(p_{t, 2}>0\right)$ is larger than $p_{t, 1}$ and the optimal income can be obtained at $p_{t, 2}$ (as shown in Figure 1), which is

$$
I_{\max }=I\left(p_{t, 2}\right)=I_{f}+\frac{\widetilde{D}_{c} e^{-\sigma\left[a+\left(1 / \sigma-v_{3} p_{s}-v_{3} p_{l} L\right)\right]}}{\left(v_{3} \sigma N\right)} .
$$

Case $2\left(p_{t, 1}>0\right.$ and $\left.p_{t, 1} \geq p_{t, 2}\right)$. In this case, the income function is characterized by two segments. The gradient of the income function regarding $p_{t}$ is larger than zero within $\left[0, p_{t, 1}\right]$, where the function is increasing. The income has a maximum value at $p_{t, 1}$ :

$$
\begin{aligned}
I_{\max }= & I\left(p_{t, 1}\right) \\
= & I_{f} \\
& +\frac{P_{c}}{t_{c}}\left(p_{s}+p_{l} L+\frac{\left[-\ln \left(D_{c m} / \widetilde{D}_{c}\right) / \sigma-a\right]}{v_{3}}\right) .
\end{aligned}
$$

Given different combinations of parameters, $p_{t, 1}$ and $p_{t, 2}$ may fall in different cases, for which the maximum incomes lie in $I\left(p_{t, 1}\right), I\left(p_{t, 2}\right)$, and $I(0)$. One can calculate the optimal income with the corresponding equation listed above. The income and demand diagrams are illustrated in Figure 2.

Case $3\left(p_{t, 1}, p_{t, 2}<0\right)$. In this case, the gradient of the income function regarding $p_{t}$ is less than 0 within $[0, \infty)$ as one can observe from (15); therefore the function is monotonously decreasing. The income has an upper bound at $p_{t}=0$, which is also the optimal income (Figure 3):

$$
I_{\max }=I(0)=I_{f}+\frac{\widetilde{D}_{c} e^{-\sigma a}\left(p_{s}+p_{l} L\right)}{N} .
$$

\subsection{Finding the Solution of Congestion Premium to a Desired Income}

Case $1\left(p_{t, 1} \leq 0 ; p_{t, 2} \geq 0\right.$; or $\left.p_{t, 2} \geq p_{t, 1}>0\right)$. In this case, the gradient of the income function regarding $p_{t}$ is larger than 0 within $\left[0, p_{t, 2}\right]$, where the function is monotonously increasing. The income has an upper bound at $p_{t, 2}$, which is also the optimal income. Figure 4 presents the feasible solutions in this case.

If $R+C>I\left(p_{t, 2}\right)$, no feasible solution exists.

If $I(0) \leq R+C<I\left(p_{t, 2}\right)$, there exist two feasible solutions.

$$
\begin{aligned}
\left(p_{s}+p_{l} L\right)+b p_{t} & =\left(\frac{N}{\widetilde{D}_{c}}\right)\left(R+C-I_{f}\right) e^{\sigma a} e^{\sigma v_{3} b p_{t}}, \\
p_{t}^{*} & =-\frac{D}{C}-\frac{1}{b} \mathscr{W}\left(\frac{A b}{C}-e^{b D / C}\right) .
\end{aligned}
$$

If $R+C<I(0)$, there exists only one feasible solution. 


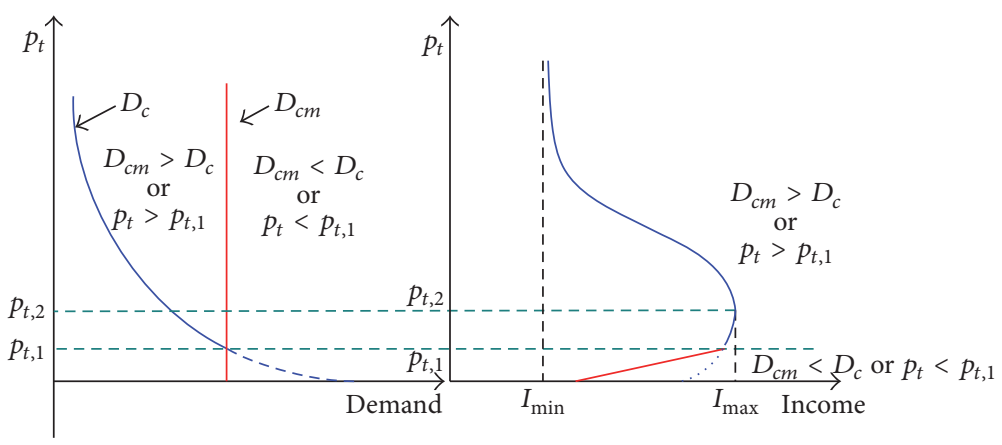

(a)

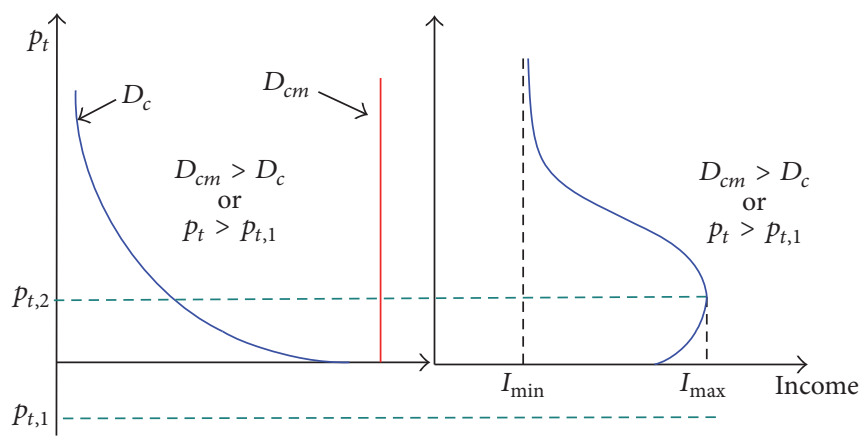

(b)

Figure 1: Two scenarios in Case 1 to demonstrate the change of the income and the demand with the raise of congestion premium: (a) the function is characterized by two points of $p_{t, 1}$ and $p_{t, 2}$; (b) the function is characterized by one point of $p_{t, 1}$.

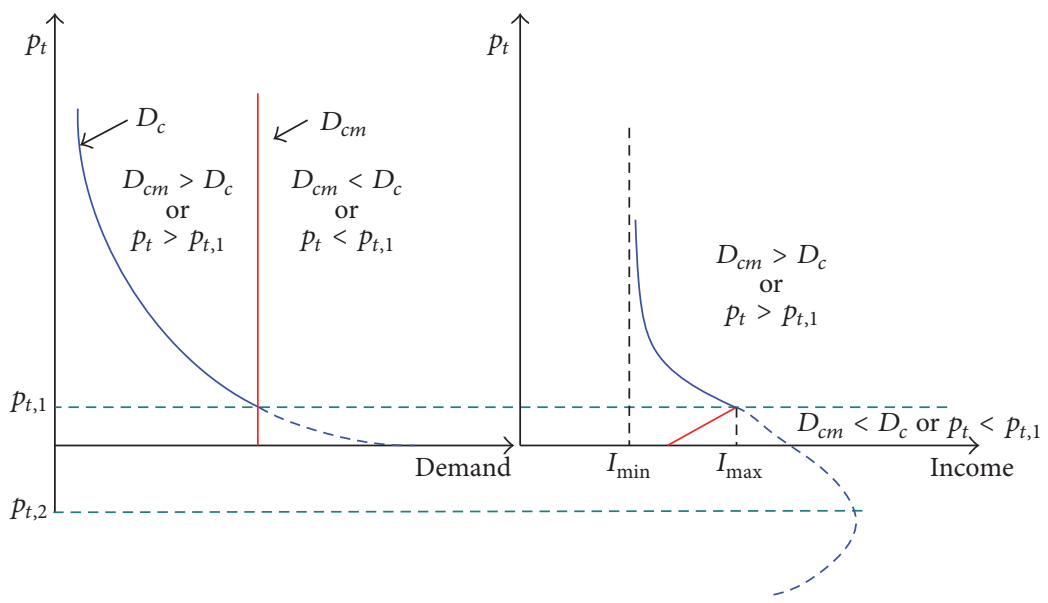

Figure 2: The diagrams to demonstrate the change of the income and the demand with the raise of congestion premium in Case 2: the function is characterized by two points of $p_{t, 1}$ and $p_{t, 2}$.

$$
\begin{aligned}
\left(p_{s}+p_{l} L\right)+b p_{t} & =\left(\frac{N}{\widetilde{D}_{c}}\right)\left(R+C-I_{f}\right) e^{\sigma a} e^{\sigma v_{3} b p_{t}} \\
p_{t}^{*} & =-\frac{D}{C}-\frac{1}{b} \mathscr{W}\left(\frac{A b}{C}-e^{b D / C}\right) .
\end{aligned}
$$

Case $2\left(p_{t, 2} \leq 0 ; p_{t, 1} \geq 0\right.$; or $\left.p_{t, 1} \geq p_{t, 2}>0\right)$. In this case, the gradient of the income function regarding $p_{t}$ is larger than 0 within $\left[0, p_{t, 1}\right]$, where the function is monotonously increasing. The income has an upper bound at $p_{t, 2}$, which is also the optimal income. Figure 5 presents the feasible solutions in this case.

If $R+C>I\left(p_{t, 1}\right)$, no feasible solution exists.

If $I(0) \leq R+C<I\left(p_{t, 1}\right)$, there exist two feasible solutions.

$$
\begin{aligned}
\left(p_{s}+p_{l} L\right)+b p_{t} & =\left(\frac{N}{\widetilde{D}_{c}}\right)\left(R+C-I_{f}\right) e^{\sigma a} e^{\sigma v_{3} b p_{t}}, \\
p_{t}^{*} & =-\frac{D}{C}-\frac{1}{b} \mathscr{W}\left(\frac{A b}{C}-e^{b D / C}\right) .
\end{aligned}
$$

If $R+C<I(0)$, there exists only one feasible solution. 


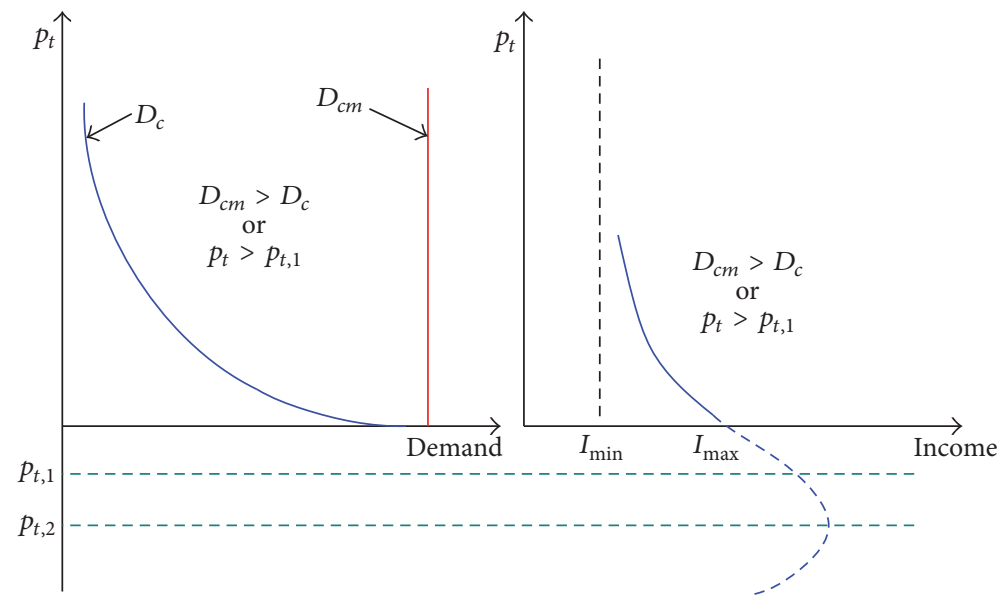

FIgURE 3: The diagrams to demonstrate the change of the income and the demand with the raise of congestion premium in Case $3\left(I_{\max }=\right.$ $I(0))$.

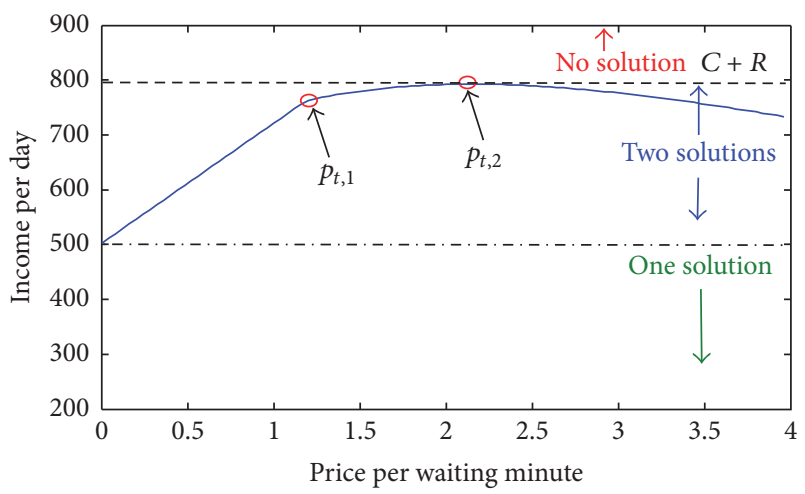

Figure 4: An illustrative diagram to demonstrate the feasible solutions in Case $1\left(\sigma=0.01 ; v_{3}=1\right)$.

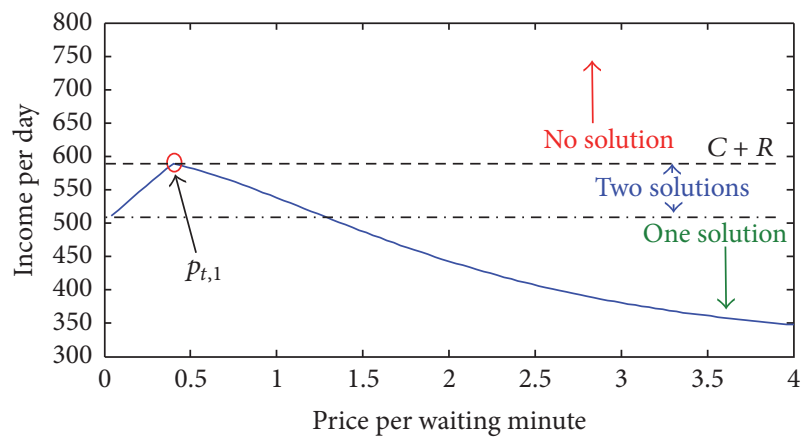

FIgURE 5: An illustrative diagram to demonstrate the feasible solutions in Case $2\left(\sigma=0.01 ; v_{3}=3\right)$.

$$
\begin{aligned}
p_{t}^{*} & =\frac{\left(R+C-I_{f}\right) t_{c} / P_{c}-p_{c}-p_{l} L}{b}, \\
\left(p_{s}+p_{l} L\right)+b p_{t} & =\left(\frac{N}{\widetilde{D}_{c}}\right)\left(R+C-I_{f}\right) e^{\sigma a} e^{\sigma v_{3} b p_{t}}, \\
p_{t}^{*} & =-\frac{D}{C}-\frac{1}{b} \mathscr{W}\left(\frac{A b}{C}-e^{b D / C}\right) .
\end{aligned}
$$

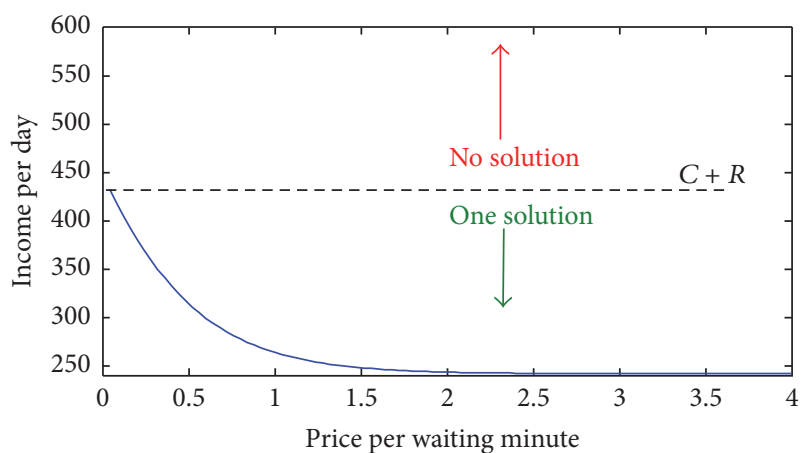

FIGURE 6: An illustrative diagram to demonstrate the feasible solutions in Case $3\left(\sigma=0.015 ; v_{3}=6\right)$.

Case $3\left(p_{t, 1}, p_{t, 2}<0\right)$. In this case, the gradient of the income function regarding $p_{t}$ is less than 0 ; therefore the function is monotonously decreasing. The income has an upper bound at $p_{t}=0$, which is also the optimal income. Figure 6 presents the feasible solutions in this case.

If $R+C>I(0)$, no feasible solution exists. Otherwise, there exists one feasible solution.

$$
\begin{aligned}
\left(p_{s}+p_{l} L\right)+b p_{t} & =\left(\frac{N}{\widetilde{D}_{c}}\right)\left(R+C-I_{f}\right) e^{\sigma a} e^{\sigma \nu_{3} b p_{t}}, \\
p_{t}^{*} & =-\frac{D}{C}-\frac{1}{b} \mathscr{W}\left(\frac{A b}{C}-e^{b D / C}\right) .
\end{aligned}
$$

\section{Beijing Case Study}

6.1. Beijing Taxi Fare Schemes. Although up to 66,700 taxicabs are serving the taxi market in Beijing, the service level of taxi has always failed to meet the expectation of the public. Service refusal, as an unpleasant phenomenon, is becoming a significant problem in Beijing's taxi market. To improve the service level in the taxi market and narrow down the gap between the supply and the demand, it is necessary to adjust the taxi fare scheme in Beijing. In this case study, we 
TABLE 1: Key parameters of the proposed model for the Beijing taxi's market.

\begin{tabular}{lc}
\hline Parameter & Value \\
\hline$\sigma$ & 1.2 \\
$v_{1}$ & 0.05 \\
$v_{2}$ & 0.1 \\
$v_{3}$ & 0.03 \\
$L / \mathrm{km}$ & 8 \\
$u_{c} / \mathrm{km} \cdot \mathrm{h}^{-1}$ & 10 \\
$u_{f} / \mathrm{km} \cdot \mathrm{h}^{-1}$ & 45 \\
$P_{c} / \mathrm{h}$ & 4 \\
$\lambda$ & 0.7 \\
\hline
\end{tabular}

applied the proposed income equilibrium model to measure the effects of different taxi fare schemes on the travel demand and the income of taxi driver. According to the taxi data from Beijing Bureau of Statistics, key parameters of the proposed model for Beijing's taxi market are summarized in Table 1 (Beijing Transportation Research Center [37] and Beijing Municipal Commission [38]).

Based on the parameters in Notations, three fare schemes are compared by applying the proposed taxi price equilibrium model, which are the original fare scheme, the current fare scheme (adjusted in June 10, 2013), and the optimal fare scheme (proposed in this paper). The relevant price indicators can be solved through the proposed model, as summarized in Table 2.

6.2. Analysis of Results. As observed from Table 1, the current fare scheme adjusted the flag-down fare, the unit mileage fare, and congestion premium comprehensively, as compared with the original one. The adjustment leads a sharp drop in the demand from 23.2 to 12.5. The decreasing rate is about $46.1 \%$. At the same time, the income also dropped from 669.9 CNY to $504.9 \mathrm{CNY}$ or decreased by $24.6 \%$ from the one in the original fare scheme. The results indicate that the current fare scheme significantly reduced the need to travel by taxi. Meanwhile, the raise of the initial fare and congestion premium cannot compensate for the loss from the decline of demand. In this regard, the decline in the revenue is too fast. This is bound to affect the level of service and the long-term healthy development of taxi market in Beijing. In a word, the current fare scheme is not an optimal one for Beijing's taxi market.

In the optimal fare scheme proposed by us, the flag-down fare and the fare per unit mileage remain the same, while congestion premium is increased from $2 \mathrm{CNY}$ to $4.6 \mathrm{CNY}$ per 5 minutes. In this case, the income is slightly increased from $669.9 \mathrm{CNY}$ to $675.9 \mathrm{CNY}$, while the demand is reduced from 23.2 to 18.9 per taxi driver per day decreasing by $18.5 \%$ from the one in the original fare scheme. The results indicate that the optimal scheme guarantees that the taxi income stays stable and restrains the demand effectively at the same time. Thus, it can promote the balance between the supply and demand in Beijing's taxi market and eventually improve the service level in the whole market.
TABle 2: Taxi demand and incomes of different price schemes in Beijing.

\begin{tabular}{lccc}
\hline Indicator & $\begin{array}{c}\text { Original } \\
\text { fare scheme }\end{array}$ & $\begin{array}{c}\text { Current fare } \\
\text { scheme }\end{array}$ & $\begin{array}{c}\text { Optimal } \\
\text { fare scheme }\end{array}$ \\
\hline$p_{s}(\mathrm{CNY})$ & 12 & 14 & 12 \\
$p_{l}(\mathrm{CNY} / \mathrm{km})$ & 2.0 & 2.3 & 2.0 \\
$p_{t}(\mathrm{CNY} / 5 \mathrm{~min})$ & 2.0 & 4.6 & 4.6 \\
$I(\mathrm{CNY})$ & 669.9 & 504.9 & 675.9 \\
$D$ & 23.2 & 12.5 & 18.9 \\
$p_{t, 1}(\mathrm{CNY} / 5 \mathrm{~min})$ & 3.2 & 1.6 & 3.2 \\
$p_{t, 2}(\mathrm{CNY} / 5 \mathrm{~min})$ & -0.033 & -0.6875 & -0.033 \\
$I_{\max }(\mathrm{CNY})$ & 722.5 & 585 & 722.5 \\
\hline
\end{tabular}

Let us reveal the reasons for the above observations. In all three fare schemes, $p_{t, 1} \geq 0$ and $p_{t, 2} \leq 0$. Thus, the solutions in Case 2 are applicable to these three schemes, as discussed in Section 5. When $p_{t} \in\left[0, p_{t, 1}\right]$, the income function is increasing and the maximum income will be obtained at $p_{t, 1}$. In the original fare scheme, $p_{t}<p_{t, 1}$, and hence the demand exceeds the maximum supply of taxi fleets. The market is served by the taxi fleet maximum capacity $\left(D_{c m}\right)$. Meanwhile in the optimal fare scheme, $p_{t}>p_{t, 1}$, and hence the demand is less than the maximum supply of taxi fleet. The equilibrium of supply and demand in the market has been reached by applying the optimal scheme. Note that the income of the optimal scheme is even slightly increased from that of the original one. The $p_{t}$-demand and the $p_{t}$-income diagrams of the two schemes are illustrated in Figure 7.

Figure 8 shows the $p_{t}$-income diagram of the current fare scheme. If $p_{t}$ is far less than $p_{t, 1}$, the demand will decrease significantly and hence the income will also drop substantially. The gains from the raise of congestion premium cannot compensate for the loss from the reduction of the demand.

As observed from the two figures (Figures 7 and 8), in the region outlined by two dashed red lines, we can find two different congestion premium fares in the vicinity of point $p_{t, 1}$ to guarantee the same income, while a higher congestion premium fare can reduce the travel demand for taxis effectively. In congested cities (e.g., Beijing), we need to reduce the excessive need for taxis to reach the equilibrium between the supply and demand in the market, while keeping the stability of the taxi driver's income to the maximum extent. In this regard, we should choose the higher value near the point of $p_{t, 1}$ as congestion premium, as illustrated in the optimal fare scheme.

\section{Conclusions}

7.1. Summaries. Traffic congestion is a growing problem in many metropolitan areas, especially in some megacities (e.g., Beijing, a city with 21 million people and 5 million vehicles). Getting stuck in congested traffic, the mileage per unit time that a taxicab travels declines significantly, and hence congestion premium becomes an increasingly important source of a taxi driver's income. In this paper, we developed 

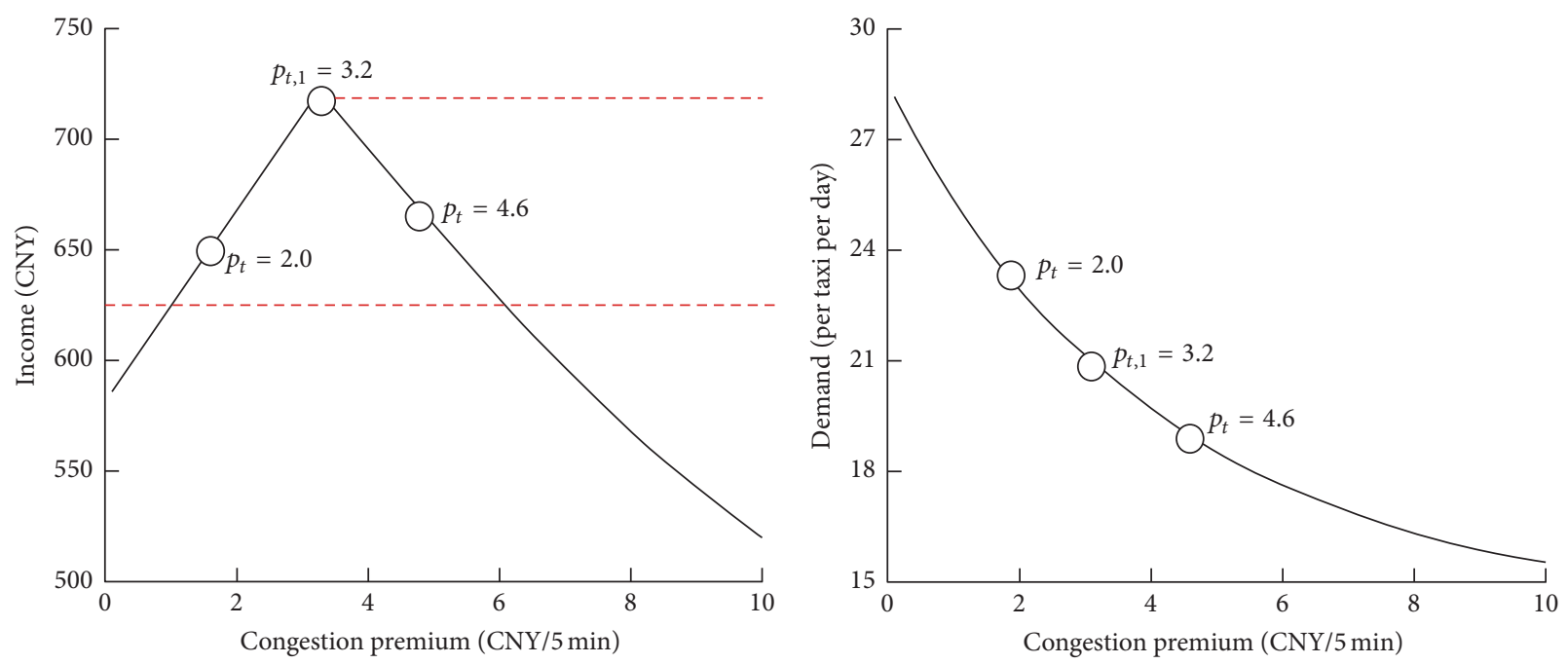

FIgURE 7: The $p_{t}$-demand and the $p_{t}$-income diagrams for the original and optimal fare schemes.

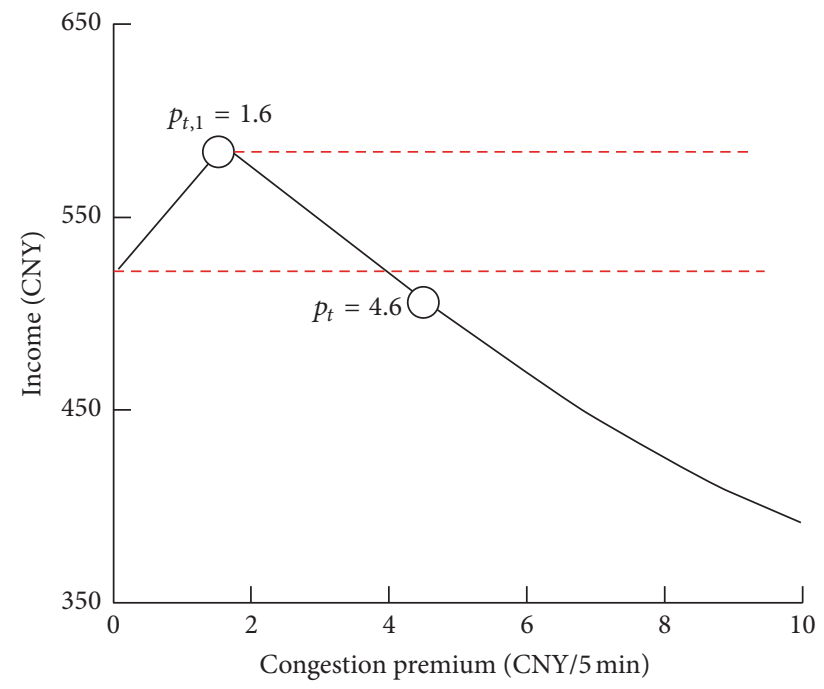

FIgURE 8: The $p_{t}$-income diagram for the current fare scheme.

a taxi price equilibrium model with congestion premium and explored the adjustment mechanism of congestion premium on optimizing the taxi driver income, balancing the supply and demand in the taxi market, and eventually improving the level of service in the whole taxi market. Two critical congestion premium fares were identified and then three cases are discussed under various scenarios with different combinations of critical congestion premium fares. A case study was provided using the real data of Beijing's taxi market to demonstrate the feasibility of the proposed model. The major findings of this study are summarized as follows:

(i) To optimize the taxi fare scheme in congested cities, two major objectives need to be considered: on one hand, the scheme can optimize or increase the taxi income and hence reduce the service refusal rate; on the other hand, the scheme can also adjust the demand effectively to reach the supply-demand equilibrium in the taxi market and hence improve the overall service level of the taxi market.

(ii) In this paper, we developed a taxi price equilibrium model with congestion premium. Two critical congestion premium fares were identified and three cases are discussed under various scenarios with different combinations of critical congestion premium fares. The process of finding the optimal solution and the solutions for a desired income were also discussed for these three cases.

(iii) The model was applied to Beijing's taxi market with the real taxi data in Beijing. The results indicated that the current taxi fare scheme (implemented in June 2013) caused the travel demand for taxi to fall by $46.1 \%$ and the income dropped by $24.6 \%$, as compared with the original one. It is apparently suboptimal, while the proposed optimal fare scheme can raise the income by 5 CNY per trip and reduce the travel demand of taxi by $18.5 \%$. The optimal one is better to build the supplydemand equilibrium of the taxi market in Beijing and hence to improve the service level.

7.2. Future Work. The current model still has some room for improvement. Some of the notable limitations and future works are summarized as follows.

Firstly, in this paper, we aim to provide a practicalenough fare equilibrium model with few parameters which is sufficient to capture the driver's profit and the customer's surplus in comparison with other more complicated models. Therefore, we used the average (or aggregated) value to capture the key variables of taxi trips, while ignoring their diverse patterns. However, this may hurt the justifiable basis of the model, since travel characteristics (i.e., speed, distance, and time) are varying for individual taxi trips. However, if we are tending to develop more realistic models, significant 
data needs will be generated due to the high number of determinants in the taxi demand and supply. In this regard, our model is a trade-off between practicality and complexity. In our future work, we will keep improving the model to make it more realistic without hurting its practical feature.

Secondly, in reality, the operation of taxi is subjected to taxi drivers' schedule. They cannot work 24 hours a day, or they have to take a break after a working shift. As a result, the realized demand $D_{r}$ is constrained not only by the maximum fleet size but also by the drivers' availability. In this regard, one of the future works for the authors is how to consider the taxi drivers' working schedule in the proposed model given the efficiency of revenue generating during off-peak and peak hours.

Lastly, recent advances in information technologies have boosted "sharing economies" in the taxicab market, where smartphone-based applications (such as Uber and Didi) that allow networked, real-time data exchange facilitate the sharing of taxi service with others. These kinds of ridesharing apps have at least two unique impacts on the traditional customer-driver search behaviors in the taxicab market: (1) the apps directly connect taxi drivers with customers looking for a ride; (2) the apps make the negotiation between customers and taxi drivers possible and provide wider choices to not only customers but also taxi drivers. Of course, negotiations are heavily involved with the fare. In this regard, the proposed approach has the potential to deal with the new ridesharing taxi service structure in nature, but the impact of this large-scale sharing on the taxicab market is still not clear and assessing its benefits quantitatively is still missing. It is definitely another future work for the authors and other taxi system researchers.

\section{Notations}

Major Variable Notations and Definitions in the Paper

p: Total ride price (CNY (the Renminbi (code: CNY) is the currency of the People's Republic of China (PRC), whose principal unit is Yuan.))

$p_{s}:$ Initial flag-fall fare (CNY)

$p_{t}$ : Congestion premium or congestion charge per hour (CNY)

$p_{l}$ : Charge per kilometer $(\mathrm{km})$

$L$ : Average length of each ride $(\mathrm{km})$

$u_{c}$ : Average traveling speed in congested network (km/hour)

$u_{f}$ : Free-flow speed (km/hour)

$u_{0}$ : Speed threshold below which the congestion based charge is activated (km/hour)

$\lambda$ : The ratio of congested sections to the overall road network

$t_{c}$ : Period when the taxi is traveling under the threshold of $u_{0}$

$t_{f}$ : Period when the taxi is traveling above the threshold of $u_{0}$
$D_{c}: \quad$ Demand per unit time (or average trip per hour)

$D_{c m}$ : Maximum number of trips which can be served by the taxi fleet

$D_{f}$ : $\quad$ Free-flow demand

$\widetilde{D}_{f}$ : $\quad$ Potential demand of free-flow trips

$D_{f r}$ : $\quad$ Realized demand in off-peak hours

$D_{c r}: \quad$ Realized demand in peak hours

$T_{c}$ : $\quad$ Average ride time

$W_{c}$ : Aggregated waiting time for taxi service

$w: \quad$ Average waiting time for a taxicab

$N$ : $\quad$ Taxi fleet size

$P_{f}: \quad$ Length of the off-peak hours

$P_{c}$ : $\quad$ Length of the peak hours

$I_{f}$ : Income during the off-peak hours

I: $\quad$ Average income of a taxi driver in both peak and off-peak hours

$c_{g}: \quad$ Fuel cost per unit of time

Q: $\quad$ License fee per day

T: $\quad$ Working hours per day

$C$ : $\quad$ Operational cost per day

$R: \quad$ Expected profit per day

$p_{t, 1} ; p_{t, 2}$ : Two critical congestion premium fares

$\sigma: \quad$ Sensitivity coefficient to the total trip cost

$v_{1}$ : Sensitivity coefficient to starting fare

$v_{2}$ : Sensitivity coefficient to the charge per length

$v_{3}$ : Sensitivity coefficient to congestion premium.

\section{Conflicts of Interest}

The authors declare that there are no conflicts of interest regarding the publication of this paper.

\section{Acknowledgments}

This study was supported by National Natural Science Foundation of China (Grant no. 51278057 and no. 51378171), Fok Ying-Tong Education Foundation, China (Grant no. 151075), and Science and Technology Program of Beijing, China (Grant no. Z121100000312101).

\section{References}

[1] C. Bian, C. Yuan, W. Kuang, and D. Wu, "Evaluation, classification, and influential factors analysis of traffic congestion in chinese cities using the online map data," Mathematical Problems in Engineering, vol. 2016, Article ID 1693729, 10 pages, 2016.

[2] D. Wei, C. Yuan, H. Liu, D. Wu, and W. Kumfer, “The impact of service refusal to the supply-demand equilibrium in the Taxicab market," Networks and Spatial Economics, pp. 1-29, 2016.

[3] C.-W. Yuan, Q.-Q. Wu, D.-L. Wei, and D.-Y. Wu, "Optimal modeling and equilibrium mechanism of taxi market with consideration of service refusal," China Journal of Highway and Transport, vol. 27, no. 6, pp. 91-97, 2014.

[4] C. Yuan, D. Wu, D. Wei, and H. Liu, A Taxi Price Equilibrium Model with the Waiting Price for Congested Cities: Beijing Case Study, 2017. 
[5] G. W. Douglas, "Price regulation and optimal service standards: the taxicab industry," Journal of Transport Economics and Policy, vol. 6, pp. 116-127, 1972.

[6] A. S. De Vany, "Capacity utilization under alternative regulatory restraints: an analysis of taxi markets," Journal of Political Economy, vol. 83, no. 1, pp. 83-94, 1975.

[7] C. F. Manski and J. D. Wright, "Nature of equilibrium in the market for taxi services," 1967.

[8] C. F. Daganzo, "An approximate analytic model of many-tomany demand responsive transportation systems," Transportation Research, vol. 12, no. 5, pp. 325-333, 1978.

[9] J. F. Foerster and G. Gilbert, "Taxicab deregulation: economic consequences and regulatory choices," Transportation, vol. 8, no. 4, pp. 371-387, 1979.

[10] R. D. Cairns and C. Liston-Heyes, "Competition and regulation in the taxi industry," Journal of Public Economics, vol. 59, no. 1, pp. 1-15, 1996.

[11] R. Arnott, "Taxi travel should be subsidized," Journal of Urban Economics, vol. 40, no. 3, pp. 316-333, 1996.

[12] J. E. L. Fernández, J. Ch. De Cea, and J. M. Briones, "A diagrammatic analysis of the market for cruising taxis," Transportation Research Part E: Logistics and Transportation Review, vol. 42, no. 6, pp. 498-526, 2006.

[13] S. K. Chang and C.-H. Chu, "Taxi vacancy rate, fare, and subsidy with maximum social willingness-to-pay under loglinear demand function," Transportation Research Record, no. 2111, pp. 90-99, 2009.

[14] M. von Massow and M. S. Canbolat, "Fareplay: an examination of taxicab drivers' response to dispatch policy," Expert Systems with Applications, vol. 37, no. 3, pp. 2451-2458, 2010.

[15] S. C. Wong and H. Yang, "Network model of urban taxi services: improved algorithm," Transportation Research Record, no. 1623, pp. 27-30, 1998.

[16] H. Yang, Y. W. Lau, S. C. Wong, and H. K. Lo, "A macroscopic taxi model for passenger demand, taxi utilization and level of services," Transportation, vol. 27, no. 3, pp. 317-340, 2000.

[17] K. Wong, S. Wong, H. Yang, and C. Tong, "A sensitivity-based solution algorithm for the network model of urban taxi services, Transportation and Traffic Theory in the 21st Century," in Proceedings of the 15th International Symposium on Transportation and Traffic Theory, pp. 23-42, Emerald Group Publishing Limited, Adelaide, Australia, July 2002.

[18] K. I. Wong, S. C. Wong, J. H. Wu, H. Yang, and W. H. K. Lam, "A combined distribution, hierarchical mode choice, and assignment network model with multiple user and mode classes," Urban and Regional Transportation Modeling: Essays in Honor of David Boyce, pp. 25-42, 2003.

[19] H. Yang, M. Ye, W. H. Tang, and S. C. Wong, "Regulating taxi services in the presence of congestion externality," Transportation Research Part A: Policy and Practice, vol. 39, no. 1, pp. 17-40, 2005.

[20] H. Yang, M. Ye, W. H.-C. Tang, and S. C. Wong, "A multiperiod dynamic model of taxi services with endogenous service intensity," Operations Research, vol. 53, no. 3, pp. 501-515, 2005.

[21] H. Yang, C. S. Fung, K. I. Wong, and S. C. Wong, "Nonlinear pricing of taxi services," Transportation Research Part A: Policy and Practice, vol. 44, no. 5, pp. 337-348, 2010.

[22] H. Yang, C. W. Y. Leung, S. C. Wong, and M. G. H. Bell, "Equilibria of bilateral taxi-customer searching and meeting on networks," Transportation Research Part B: Methodological, vol. 44, no. 8-9, pp. 1067-1083, 2010.
[23] T. Yang, H. Yang, and S. Wong, "Modeling taxi services with a bilateral taxi-customer searching and meeting function," in Proceedings of the Annual Meeting of Transportation Research Board, Transportation Research Board (TRB), 2010.

[24] K. Wong, S. Wong, and H. Yang, "Calibration and validation of network equilibrium taxi model for Hong Kong," in Transportation Science and Technology into the Next Millenium: Proceedings of the Fourth Conference of the Hong Kong Society for Transportation Studies, Hong Kong, HKUST, Hong Kong, 1999.

[25] H. Yang and S. C. Wong, "A network model of urban taxi services," Transportation Research Part B: Methodological, vol. 32, no. 4, pp. 235-246, 1998.

[26] D. Wei, F. Chen, and X. Sun, "An improved road network partition algorithm for parallel microscopic traffic simulation," in Proceedings of the International Conference on Mechanic Automation and Control Engineering (MACE '10), pp. 27772782, Wuhan, China, June 2010.

[27] H. Yang, S. C. Wong, and K. I. Wong, "Demand-supply equilibrium of taxi services in a network under competition and regulation," Transportation Research Part B: Methodological, vol. 36, no. 9, pp. 799-819, 2002.

[28] D. Wei and H. Liu, "An adaptive-margin support vector regression for short-term traffic flow forecast," Journal of Intelligent Transportation Systems: Technology, Planning, and Operations, vol. 17, no. 4, pp. 317-327, 2013.

[29] D. Wei and H. Liu, "Analysis of asymmetric driving behavior using a self-learning approach," Transportation Research Part B: Methodological, vol. 47, pp. 1-14, 2013.

[30] C. Kamga, M. A. Yazici, and A. Singhal, "Analysis of taxi demand and supply in New York City: implications of recent taxi regulations," Transportation Planning and Technology, vol. 38, no. 6, pp. 601-625, 2015.

[31] X. Zhan, X. Qian, and S. V. Ukkusuri, "Measuring the efficiency of urban taxi service system," in Proceedings of the 3rd International Workshop on Urban Computing (UrbComp '14), pp. 1-9, New York, NY, USA, 2014.

[32] V. Salnikov, R. Lambiotte, A. Noulas, and C. Mascolo, "OpenStreetCab: exploiting taxi mobility patterns in New York City to reduce commuter costs," https://arxiv.org/abs/1503.03021.

[33] D. Wei, F. Chen, and T. Zhang, "Least square-support vector regression based car-following model with sparse sample selection," in Proceedings of the 8th World Congress on Intelligent Control and Automation (WCICA '10), pp. 1701-1707, July 2010.

[34] D. Wei, F. Chen, and C. Chen, "Wavelet support vector regression for short-term traffic forecasting," Mediterranean Journal of Measurement and Control, vol. 6, no. 2, pp. 39-45, 2010.

[35] C. Yuan, D. Wei, and H. Liu, "The impact of service refusal to the supply-demand equilibrium in taxicab market," in Proceedings of the 93rd Annual Meeting of the Transportation Research Board, January 2014.

[36] K. I. Wong, S. C. Wong, and H. Yang, "Modeling urban taxi services in congested road networks with elastic demand," Transportation Research Part B: Methodological, vol. 35, no. 9, pp. 819-842, 2001.

[37] Beijing Transportation Research Center, Annual Report of Transportation of Beijing, 2011.

[38] “2013 Report of Public Transport Beijing," Beijing Municipal Commission of Transport. 


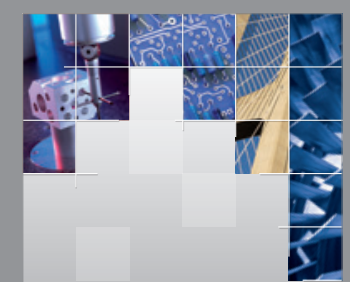

\section{Enfincering}
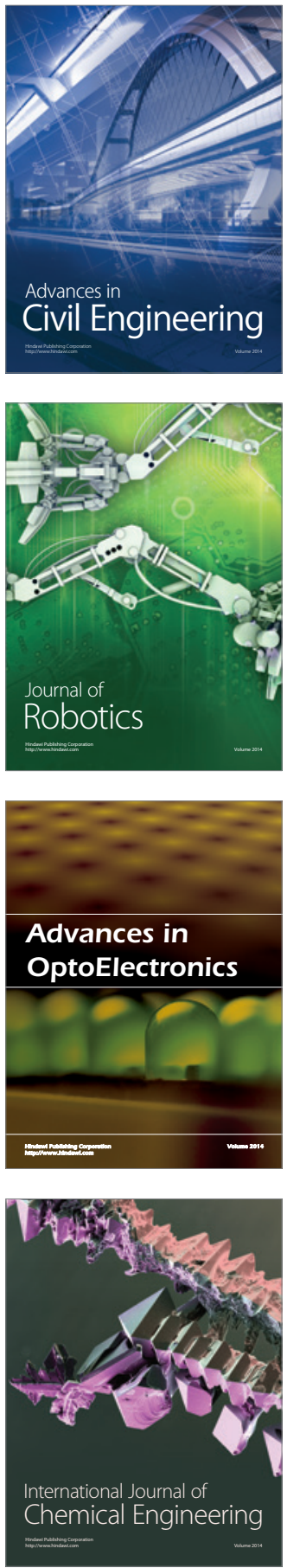

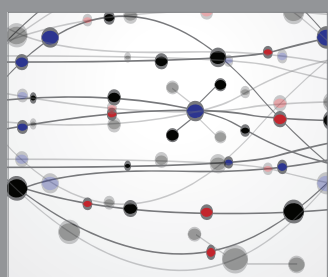

The Scientific World Journal

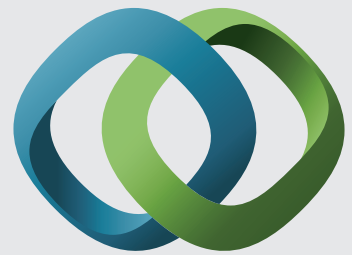

\section{Hindawi}

Submit your manuscripts at

https://www.hindawi.com
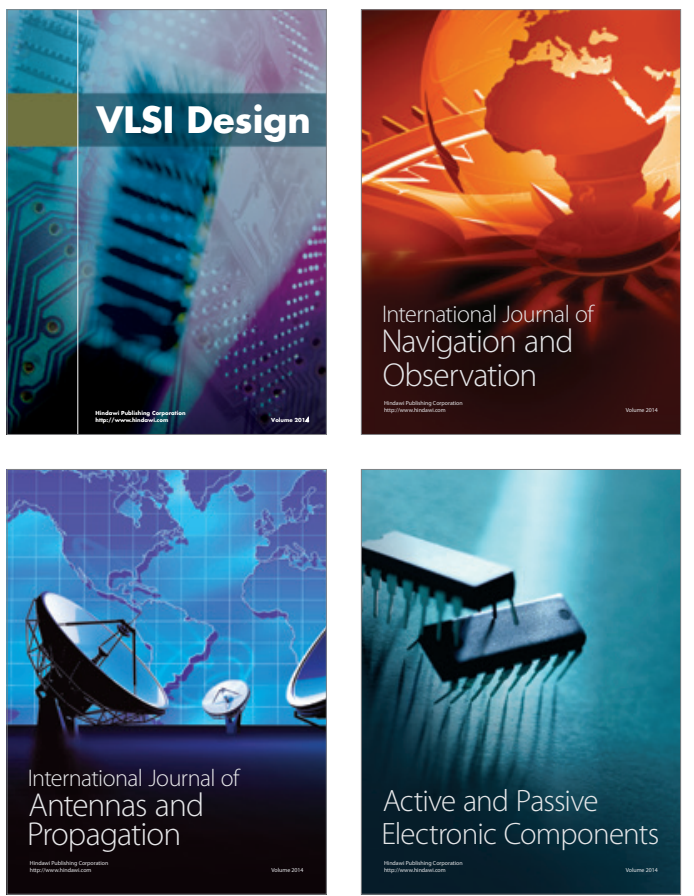
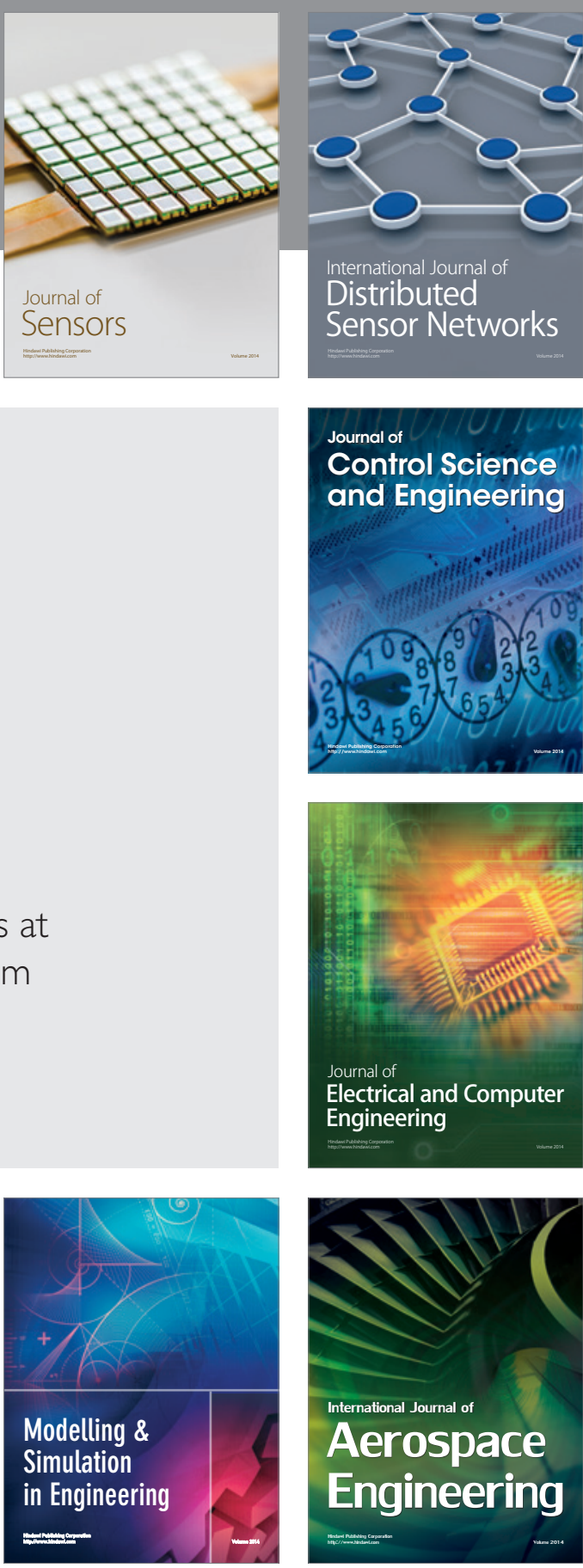

International Journal of

Distributed

Sensor Networks

$-$

Joumal of

Control Science

and Engineering
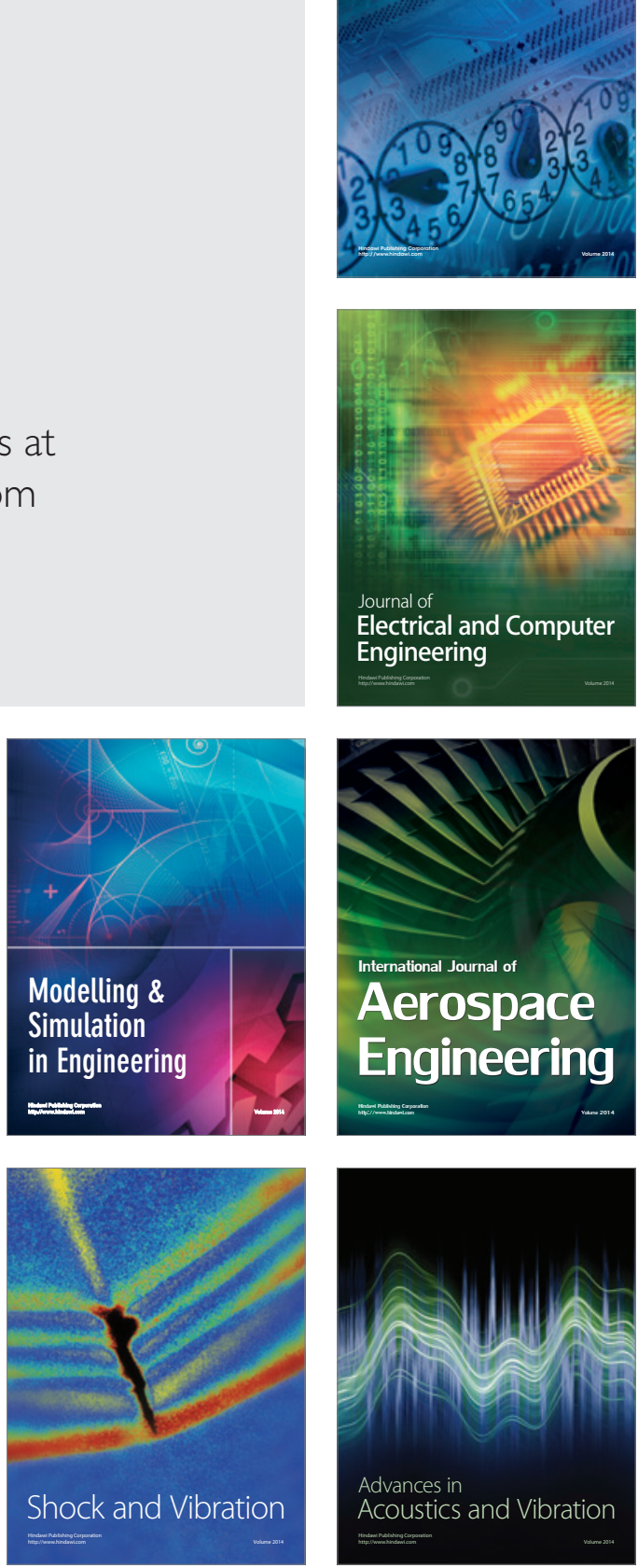